\title{
Value of T1 Mapping on Gadoxetic acid-enhanced MRI for Microvascular Invasion of Hepatocellular Carcinoma: A Retrospective Study
}

chenyi Rao

Nantong University Medical School

Xinquan Wang

Affiliated Hospital of Nantong University

Minda Li

Affiliated Hospital of Nantong University

Guofeng Zhou

Zhongshan Hospital Fudan University

Hongmei Gu ( $\nabla$ raoxray@126.com )

Affiliated Hospital of Nantong University

Research article

Keywords: T1 mapping, hepatocellular carcinoma, microvascular invasion, magnetic resonance imaging

Posted Date: January 2nd, 2020

DOI: https://doi.org/10.21203/rs.2.19955/v1

License: (c) (i) This work is licensed under a Creative Commons Attribution 4.0 International License.

Read Full License

Version of Record: A version of this preprint was published at BMC Medical Imaging on April 28th, 2020.

See the published version at https://doi.org/10.1186/s12880-020-00433-y. 


\section{Abstract}

Background: To evaluate the utility of non-invasive parameters driving from T1 mapping on gadoxetic acid-enhanced MRI for predicting microvascular invasion (MVI) of hepatocellular carcinoma (HCC) compared with diffusion-weighted imaging (DWI).

Methods: A total of 94 patients with single HCC undergoing partial hepatectomy was included in the retrospective study, who underwent preoperative gadoxetic acid-enhanced MRI combined with DWI and T1 mapping. Parameters including precontrast, postcontrast and reduction rate of T1 relaxation time and $A D C$ values were measured for differentiating MVI-positive HCCs $(n=38)$ from MVI-negative HCCs $(n=56)$. The receiver operating characteristic curve (ROC) was analyzed to compare the diagnostic performance of the calculated parameters.

Results: The mean value of postcontrast T1 relaxation time were significantly higher in MVI-positive HCCs that MVI-negative HCCs (621.0 vs. 536.5, $P<0.001$ ). MVI-positive HCCs demonstrated significantly lower reduction rates of T1 relaxation time and lower ADC values than MVI-negative HCCs (39.4\% vs 49.9, $P<0.001 ; 1.495 \times 10^{-3} \mathrm{~mm}^{2} / \mathrm{s}$ vs $1.620 \times 10^{-3} \mathrm{~mm}^{2} / \mathrm{s}, P=0.003$, respectively). The area under receiver operating characteristic curves were $0.587,0.728,0.824$ and 0.690 for precontrast, postcontrast, reduction rate of $\mathrm{T} 1$ relaxation time and $\mathrm{ADC}$, respectively. The reduction rate of $\mathrm{T} 1$ relaxation time was the most reliable feature with sensitivity, specificity and accuracy of the cut-off value (44.9\%) of $79.0 \%$, $73.2 \%, 75.5 \%$, respectively.

Conclusions: Reduction rate of T1 relaxation time on gadoxetic acid-enhanced MRI holds promise for evaluating MVI status of HCC.

\section{Background}

Hepatocellular carcinoma (HCC) ranks the sixth most frequent cancer and the fourth leading cause of cancer-related death worldwide in 2018 [1]. Liver resection, liver transplantation and radiofrequency ablation are the curative treatment modalities for $\mathrm{HCC}$ with different indications [2]. However, early recurrence is a major problem that impairs patient prognosis and microvascular invasion (MVI) of $\mathrm{HCC}$ is a critical predictor for early recurrence and poor prognosis after curative treatments [3-5]. Non-invasive evaluation of MVI before surgery is important as it allows for optimal treatment modalities $[4,5]$.

In clinical practice, $\mathrm{MVI}$ is determined by pathologic evaluation on the surgical specimens after liver resection or transplantation. There are some studies that demonstrating promising results for preoperatively predicting MVI of HCC based on qualitative analysis of morphologic MR imaging features $[6,7]$ and quantitative analysis including apparent diffusion coefficient (ADC) values of diffusion weighted imaging (DWI) [8,9], kurtosis value of diffusion kurtosis imaging [10] or D value of intravoxel incoherent motion [11]. Recently, a radiomics approach based on radiological images [12] provided satisfactory diagnostic performance for preoperative evaluation of MVI. However, the subjective nature of the evaluation of morphologic features, instable image quality of diffusion weighted/kurtosis images 
(i.e., T2 blackout effect, susceptibility artifacts and image distortion) or obscure algorithms of radiomics analysis are challenges for further clinical utility.

Gadoxetic acid-enhanced magnetic resonance imaging (Gd-EOB-DTPA MRI) is accepted widely as a preferred imaging method for detection and stage of hepatic nodules [2], and evaluation of liver function in patients with HCC [13]. The signal intensity (SI) on the hepatobiliary phase (HBP) of gadoxetic acidenhanced MRI determined by the mount of Gd-EOB-DTPA uptake by HCC was associated with tumor invasiveness and clinical outcome $[14,15]$. T1 mapping is an absolute and more reliable value than SI measurement for reflecting the Gd-EOB-DTPA uptake within tissue [16]. Additionally, T1 mapping with high temporal and spatial resolution images could integrate seamlessly into gadoxetic acid-enhanced MRI scan protocol. Previous studies demonstrated that gadoxetic acid-enhanced MRI combined with T1 mapping had potential for predicting histologic grades and recurrence after resection of HCC [17-19]. It supposed that the measurement of T1 relaxation time is expected to predict MVI of HCC.

Thus, the aim of our study was to compare the diagnostic performance between parameters driving from T1 mapping and DWI on gadoxetic acid-enhanced MRI in evaluation of MVI of HCC.

\section{Methods}

This study was approved by the Institutional Review Board of Zhongshan Hospital, Fudan University (approval number B2018-236) in accordance with the ethical guidelines of the Declaration of Helsinki. The committee waived the requirement for informed consent because it is a retrospective study.

\section{Patients selection}

According to the AASLD guideline, patients with preexisting cirrhosis were at high risk for developing HCC and surveillance program of ultrasound (US) and a-fetoprotein (AFP) were recommended. In our institution, between February 2016 and March 2017, 222 patients underwent gadoxetic acid-enhanced MRI for further evaluation of suspicious HCCs detected during surveillance. Patients with probable benign nodules (i.e., cysts, hemangiomas, arterioportal shunts) screened by US or patients with HCCs having any previous treatments such as transcatheter arterial chemoembolization (TACE) and radiofrequency ablation (RFA) were initially excluded from the study. The inclusive criteria of the patient selection were: (a) single HCC with histology and preoperative gadoxetic acid-enhanced MRl; (b) the interval time between MRI protocol and the operation was less than 2 weeks; (c) patients in Child-Pugh A-B; (d) HCC with qualified MR images. Of the 222 patients, 55 patients were excluded for having two or more HCCs; 8 patients were excluded for Child-Pugh C; 28 were excluded for other types of nodules including intrahepatic cholangiocarcinoma $(n=15)$, combined HCC and cholangiocarcinoma $(n=1)$, dysplastic nodule $(n=10)$ and metastasize $(n=2) ; 16$ patients were excluded for more than 2 weeks interval time during follow-up; 21 patients were excluded for lesions on left lobe with susceptibility or respiratory motion artifacts on DW images. Finally, 94 patients with single HCC were included in our study (Fig. 1). 


\section{MR Imaging Protocol}

All patients enrolled in our study underwent gadoxetic acid-enhanced MRI in a single 1.5-T MR system (MAGNETOM Aera, Siemens Healthcare, Erlangen, Germany), with a 8-channel phased-array receiver coil. Single-spin echo plane DWI for free breathing (3200/56 milliseconds repetition time (TR) / echo time (TE), $84 \times 128$ matrix, 380-400 $\times 300-324 \mathrm{~mm}$ field of view (FOV), $5.5 \mathrm{~mm}$ slice thickness) was performed, and corresponding ADC maps were automatically generated with $b$ values of 0 and $500 \mathrm{~s} / \mathrm{mm}^{2}$. Dynamic contrast-enhanced T1-weighted 3D gradient-recalled echo images (3.47 / 1.36 TR / TE, 320 × 195 matrix, $10^{\circ}$ flip angle, $308 \times 380 \mathrm{~mm}$ FOV, $3 \mathrm{~mm}$ slice thickness) were obtained after intravenously injection of contrast agent. A dual flip-angle (Flip angle, $2^{\circ}$ and $12^{\circ}$ ) before and at $20 \mathrm{~min}$ after injection of gadoxetic acid based on a voxel-by-voxel basis was applied for generating T1 maps with syngo Maplt. The precontrast phase was obtained before a bolus injection of $0.025 \mathrm{mmol} / \mathrm{kg}$ of gadoxetic acid (Primovist, Bayer Schering Pharma, Berlin, Germany) at a rate of $1 \mathrm{ml} / \mathrm{sec}$ with a subsequent $20 \mathrm{ml}$ saline flush. Subsequent MR images during the arterial phase (automatically triggered when the ascending aorta reached peak enhancement), the portal vein phase (about 14 seconds), the transition phase (about 3 minutes), and the hepatobiliary phase (HBP; 20 minutes) were obtained.

\section{MR images analysis}

Two abdominal radiologists (Blinded) independently reviewed the MR images of all the patients. Region

of interest (ROI) was outlined around the edge of tumor on each slice on precontrast T1 maps, postcontrast T1 maps and ADC maps, which were copied from ROls of HCCs that drawn on high flip angle $\left(12^{\circ}\right)$ T1-weighted images and high b value images $\left(500 \mathrm{~s} / \mathrm{mm}^{2}\right)$, respectively (Fig. 2). Precontrast, postcontrast $\mathrm{T} 1$ relaxation time and $\mathrm{ADC}$ values of the two observers were then calculated. We also calculated the reduction rate $(\Delta \%)$ of T1 relaxation time using the following formula: $\Delta \%=100 \% \times($ pre T1 value - post T1 value) / pre T1 value, in which pre T1 and post T1 values representing the T1 relaxation times before and after injection of gadoxetic acid.

\section{Reference standard for MVI}

Pathological data including presence of cirrhosis, Edmondson-Steiner grade or microvascular invasion was according to surgical pathologic reports generated by our institutional pathologists specialized in liver histology (each individual with more than 20 years of experience). Microvascular invasion was defined as presence of muscular wall in any vascular space with invasion or adherence of any intravascular tumors to the vessel wall visible microscopically.

\section{Statistical Analysis}

Frequencies of categorical variables for differentiating MVI were co mpared by using Fisher exact test. Difference of quantitative variables including precontrast/postcontrast T1 relaxation time and ADC 
values between MVI-positive and MVI-negative groups was compared by using independent sample t test. The interclass correlation coefficient (ICC) of quantitative data between the two observers was calculated (poor: <0.40; fair: 0.40-059; good: 0.60-0.74; excellent: 0.75-1.00). Area under receiver operating characteristic curve (AUC) with $95 \%$ confidence interval $(95 \% \mathrm{Cl})$ based on receiver operating characteristic curve (ROC) analysis was generated for evaluating the utility of variables to discriminate the status of MVI. Sensitivity, specificity, accuracy, positive predictive value (PPV), negative predictive value (NPV) and likelihood ratio (LR) of appropriate cutoff value corresponding to maximal Youden index by using ROC analysis were presented with $95 \% \mathrm{Cl}$. All the statistical tests were performed by using statistical software (SPSS version 21, SPSS, Chicago, III) and a two-side P value less than 0.05 indicating significance level.

\section{Results}

\section{Patients and treatment characteristics}

A total of 94 patients (76 men and 18 women; median age: 54 years (range, 24-75 years)) with single HCC were included in the study. Among the 94 patients, 87 of them (92.6\%) were hepatitis B virus infected. The mean size of maximal diameter of tumors estimated on HBP was $1.9 \mathrm{~cm}$, ranging from 1.2 to $4.5 \mathrm{~cm}$. All the patients underwent partial hepatectomy for HCC. Fifty-six HCCs were categorized of presence of MVI and $38 \mathrm{HCCs}$ were absence of MVI confirmed by histology. The other main characteristics of patients are shown in Table 1.

\section{T1 relaxation time and $\mathrm{ADC}$ measurements}

Table 2 shows the mean values of T1 relaxation time (pre-contrast, postcontrast) and ADC of the two readers. The reduction rate was then calculated based on the mean values of the two readers. There was no significant difference for precontrast T1 relaxation time between MVI-negative and MVI-positive groups (Figure 3). The mean values of postcontrast T1 relaxation time of the two readers were significantly higher in MVI-positive HCCs compared with MVI-negative HCCs $(621.0 \mathrm{~ms}$ vs $536.5 \mathrm{~ms}$, $P<0.001)$. The reduction rates of T1 relaxation time was significantly lower in MVI-positive HCCs than in MVI-negative HCCs (39.4\% vs 49.9, $P<0.001$, Figure 4). The mean values ADC value was significantly lower in MVI-positive HCCs than MVI-negative HCCs $\left(1.495 \times 10^{-3} \mathrm{~mm}^{2} / \mathrm{s}\right.$ vs $\left.1.620 \times 10^{-3} \mathrm{~mm}^{2} / \mathrm{s}, P=0.003\right)$. The agreements between two readers shown in Table 2 were excellent for ADC, precontrast and postcontrast T1 relaxation time (ICC: $0.759,95 \% \mathrm{Cl}$ : $0.637-0.840$; ICC: $0.823,95 \% \mathrm{Cl}$ : $0.744-0.879$; ICC: 0.858, 95\% Cl: 0.786-0.906, respectively).

\section{Diagnostic performance for evaluating MVI of HCC}

The ROC curves with AUC were analyzed to compare the diagnostic performance of the parameters driving from $T 1$ relaxation time and ADC of DWI for evaluation of MVI status of HCC (Figure 5). The corresponding AUC, cutoff value, sensitivity, specificity, +LR, -LR, PPV and NPV with $95 \% \mathrm{Cl}$ are summarized in Table 3. AUCs were $0.587,0.728,0.824$ and 0.690 for precontrast, postcontrast, reduction 
rate of $\mathrm{T} 1$ relaxation time and $\mathrm{ADC}$, respectively. Among the parameters of $\mathrm{T} 1$ relaxation time and $\mathrm{ADC}$, the reduction rate was the most reliable feature with an AUC of 0.824 (95\% Cl: $0.732-0.895)$, and the sensitivity, specificity and accuracy of the cut-off value (44.9\%) were $79.0 \%, 73.2 \%, 75.5 \%$, respectively.

The AUC of reduction rate of T1 relaxation time was significantly higher than that of ADC $(0.824 \mathrm{vs} 0.690$, $P=0.043)$.

\section{Discussion}

The study demonstrated that HCC with MVI had significantly higher postcontrast and reduction rate of T1 relaxation time than $\mathrm{HCC}$ without MVI. The reduction rate of T1 relaxation time demonstrated a better diagnostic performance for predicting MVI status of HCC in comparison with ADC.

Gadoxetic acid-enhanced MRI combined with DWI is a part of the standard workup in detection and characterization of hepatic nodules for better providing clinicians with roadmap of therapeutic strategies in our institution. Considering the pitfalls of DWI sequence and subjective nature of evaluating morphological imaging features on gadoxetic acid-enhanced MRI for assessing MVI, we quantitatively compared parameters deriving from T1 relaxation time of HCC with values of ADC maps that demonstrating good image quality for identifying the MVI status of HCC. Previous studies reported that $A D C$ values based on DWI was useful for evaluation of $M V I$ status of $\operatorname{HCC}[8,9,20]$. In line with our study, lower ADC value has been proven helpful for predicting MVI of HCC because it reflects higher tissue cellularity and decreased micro-capillary perfusion [9]. There are several pitfalls of DWI that affect the reliability of the $A D C$ measurement including [21, 22]: (1) limited image quality with poor signal-to-noise ratio and low spatial resolution; (2) more sensitive to motion and air susceptibility, especially for pulsation artifacts in left robe; (3) misregistration artifacts on ADC map; (4) T2 blackout effect mainly refer to fibrotic tissues or calcifications depicting hypointesity on both DW images and ADC maps. Some studies reported that the reproducibility for ADC and IVIM measurement of hepatic nodules was poor [23-25].

T1 mapping on gadoxetic acid-enhanced MRI can be used as an additional protocol included in the routine MR sequences for evaluation of diffuse liver disease [13, 26-27]. Ding [26] et al reported that the measurement of $\mathrm{T} 1$ relaxation time parameters was more reproducible compared with the measurement of ADC values for staging hepatic fibrosis. Previous studies also showed T1 mapping on gadoxetic acidenhanced MRI outperformed DWI for evaluation of liver function in patients with HCC [13] and staging hepatic fibrosis [26, 27].

The reduction rate of $\mathrm{T} 1$ relaxation time demonstrated improved diagnostic performance compared with $A D C$ values after excluding the cases that showing moderate to evident artifacts of tumor on DW images. In our institution, the sequence of T1 mapping with Syngo Maplt is routinely performed, which can provide the acquisition of MR images with high resolution 3D-dataset of whole liver. Our results demonstrated that there was no significant value in predicting MVI of HCC by using precontrast T1 relaxation time because it may be affected by some factors such as liver inflammation [28]. Our results 
indicated that lower reduction rate of T1 relaxation time was a potential predictor for MVI positive status of HCC. Peng [17] et al reported that the reduction rate in T1 value was the best predictor correlated with degree of differentiation of HCC, and higher histological grade is correlated with MVI positive status of HCC [29]. Wang [18] et al demonstrated that reduction rate of T1 relaxation time was a reliable biomarker

for predicting recurrence of $\mathrm{HCC}(\leq 3 \mathrm{~cm})$ after hepatectomy. The SI of hepatobiliary phase on gadoxetic acid-enhanced MRI has shorten T1 effect that determined by expression levels of the organic anion transporter 1B3 (OATP8) protein in HCC, and was reported to have a strong association with the expression of Wnt/ $\beta$-catenin target genes [30]. Additionally, $\beta$-catenin gene (CTNNB1) mutations of HCC showed more aggressive tumor biology with increased probability of MVI [31]. Hence, MVI positive HCC may show higher SI on HBP and lower reduction rate of T1 relaxation time.

The present study is limited by its selection bias of retrospective nature. The sample size of the study is relative small. Additionally, we only use two b-values of $0,500 \mathrm{~s} / \mathrm{mm}^{2}$ for DW imaging that routinely performed in our institution. The measurement of $A D C$ values based on these two $b$ values was previously applied for evaluating MVI of small HCC with satisfactory diagnostic performance [20]. In our study, to reduce the measurement error, we excluded DW images with moderate to evident artifacts, especially those showing artifacts caused by pulsation artifacts in the left lobe, misregistration and air susceptibility.

\section{Conclusions}

Currently, MVI of HCC can only be confirmed by histology and noninvasive approach to MVI status of HCC for guiding tumor management, such as selecting appropriate allocation of liver transplantation [4] and resection margin [5] is limited. So far, there is a large body of evidence that gadoxetic acid-enhanced MRI combined with DWI and T1 mapping can show high accuracy for characterization of focal liver lesions and evaluation of the whole and segmental liver function reserve. ADC value of DWI helps improve the diagnostic accuracy of MVI of HCC [2], however, the reliability of measurement can also be affected by technical factors. Our results suggested that T1 relaxation time measurement on gadoxetic acid-enhanced MRI holds promise to provide additional information for HCC MVI status, which is preliminary and warrants further validation.

\section{Declarations}

\section{Ethics approval and consent to participate:}

This study was approved by the Institutional Review Board of Zhongshan Hospital, Fudan University (approval number B2018-236) in accordance with the ethical guidelines of the Declaration of Helsinki. The committee waived the requirement for informed consent because it is a retrospective study.

Consent for publication: Not applicable.

Competing interests: no conflict of interest. 
Funding: This research did not receive any specific grant from funding agencies.

\section{Authors' contributions:}

All authors have read and approved the manuscript. HMG designed and supervised the study; CYR, GFZ, XQW collected the patient's clinical and MRI data; MDL, GFZ analyzed the data; CYR drafted the paper.

Acknowledgements: Not applicable

\section{Availability of data and materials:}

The datasets used and/or analysed during the current study are available from the corresponding author on reasonable request.

\section{Abbreviations}

MVI: microvascular invasion; HCC: hepatocellular carcinoma;

DWI: diffusion-weighted imaging; ROC: receiver operating characteristic curve;

ADC: apparent diffusion coefficient; DWl: diffusion weighted imaging;

Gd-EOB-DTPA MRI: Gadoxetic acid-enhanced magnetic resonance imaging;

HBP: hepatobiliary phase; US: ultrasound; AFP: a-fetoprotein;

TACE: transcatheter arterial chemoembolization; RFA: radiofrequency ablation;

TR: repetition time; TE echo time; FOV: field of view;

ROI: Region of interest; ICC: interclass correlation coefficient;

AUC: Area under receiver operating characteristic curve;

ROC: receiver operating characteristic curve;

PPV: positive predictive value; NPV: negative predictive value;

LR: likelihood ratio; organic anion transporter: OATP8;

\section{References}

1. Bray F, Ferlay J, Soerjomataram I, Siegel RL, Torre LA, Jemal A. Global cancer statistics 2018: GLOBOCAN estimates of incidence and mortality worldwide for 36 cancers in 185 countries. CA Cancer J Clin. 2018;68(6):394-424. doi: 10.3322/caac.21492. 
2. Omata M, Cheng AL, Kokudo N,et al. Asia-Pacific clinical practice guidelines on the management of hepatocellular carcinoma: a 2017 update. Hepatol Int. 2017;11(4):317-370. doi: 10.1007/s12072017-9799-9.

3. Lim KC, Chow PK, Allen JC,et al. Microvascular invasion is a better predictor of tumor recurrence and overall survival following surgical resection for hepatocellular carcinoma compared to the Milan criteria. Ann Surg. 2011;254(1):108-13. doi: 10.1097/SLA.0b013e31821ad884

4. Vitale A, Huo TL, Cucchetti A, et al. Survival Benefit of Liver Transplantation Versus Resection for Hepatocellular Carcinoma: Impact of MELD Score. Ann Surg Oncol. 2015;22(6):1901-7. doi: 10.1245/s10434-014-4099-2.

5. Han J, Li ZL, Xing H, et al. The impact of resection margin and microvascular invasion on long-term prognosis after curative resection of hepatocellular carcinoma: a multi-institutional study. HPB (Oxford). 2019;21(8):962-971. doi: 10.1016/j.hpb.2018.11.005.

6. Kim MJ, Lee M, Choi JY, Park YN. Imaging features of small hepatocellular carcinomas with microvascular invasion on gadoxetic acid-enhanced MR imaging. Eur J Radiol. 2012;81(10):2507-12. doi: 10.1016/j.ejrad.2011.11.014.

7. Lee S, Kim SH, Lee JE, Sinn DH, Park CK. Preoperative gadoxetic acid-enhanced MRI for predicting microvascular invasion in patients with single hepatocellular carcinoma. J Hepatol. 2017;67(3):526534. doi: 10.1016/j.jhep.2017.04.024.

8. Yang $\mathrm{C}$, Wang $\mathrm{H}$, Tang Y,et al. ADC similarity predicts microvascular invasion of bifocal hepatocellular carcinoma. Abdom Radiol (NY). 2018;43(9):2295-2302. doi: 10.1007/s00261-018$1469-4$.

9. Suh YJ, Kim MJ, Choi JY, Park MS, Kim KW. Preoperative prediction of the microvascular invasion of hepatocellular carcinoma with diffusion-weighted imaging.Liver Transpl. 2012;18(10):1171-8. doi: 10.1002/It.23502.

10. Wang WT, Yang L, Yang ZX,et al. Assessment of Microvascular Invasion of Hepatocellular Carcinoma with Diffusion Kurtosis Imaging. Radiology. 2018;286(2):571-580. doi: 10.1148/radiol.2017170515.

11. Wei Y, Huang Z, Tang H,et al. IVIM improves preoperative assessment of microvascular invasion in HCC. Eur Radiol. 2019;29(10):5403-5414. doi: 10.1007/s00330-019-06088-w.

12. Ma X, Wei J, Gu D,et al. Preoperative radiomics nomogram for microvascular invasion prediction in hepatocellular carcinoma using contrast-enhanced CT. Eur Radiol. 2019;29(7):3595-3605. doi: 10.1007/s00330-018-5985-y.

13. Ding Y, Rao SX, Chen C, Li R, Zeng MS. Assessing liver function in patients with HBV-related HCC: a comparison of T囚 mapping on Gd-EOB-DTPA-enhanced MR imaging with DWI. Eur Radiol. 2015;25(5):1392 May -8. doi: 10.1007/s00330-014-3542-x.

14. Yamashita T, Kitao A, Matsui O, et al. Gd-EOB-DTPA-enhanced magnetic resonance imaging and alpha-fetoprotein predict prognosis of early-stage hepatocellular carcinoma. Hepatology. 2014 Nov;60(5):1674-85. doi: 10.1002/hep.27093. 
15. Ariizumi SI, Ban D, Abe Y, et al. High-signal-intensity MR Image in the Hepatobiliary Phase Predicts Long-term Survival in Patients With Hepatocellular Carcinoma.Anticancer Res. 2019 Aug;39(8):42194225. doi: 10.21873/anticanres.13583.

16. Haimerl M, Verloh N, Zeman F,et al. Gd-EOB-DTPA-enhanced MRI for evaluation of liver function: Comparison between signal-intensity-based indices and T1 relaxometry. Sci Rep. 2017;7:43347. doi: $10.1038 /$ srep43347.

17. Peng Z, Jiang $M$, Cai H,et al. Gd-EOB-DTPA-enhanced magnetic resonance imaging combined with T1 mapping predicts the degree of differentiation in hepatocellular carcinoma. BMC Cancer. 2016;16:625. doi: 10.1186/s12885-016-2607-4.

18. Wang WT, Zhu S, Ding Y, et al. T1 mapping on gadoxetic acid-enhanced MR imaging predicts recurrence of hepatocellular carcinoma after hepatectomy. Eur J Radiol. 2018 Jun;103:25-31. doi: 10.1016/j.ejrad.2018.03.027.

19. Qin X, Yang T, Huang Z, et al. Hepatocellular carcinoma grading and recurrence prediction using T1 mapping on gadolinium-ethoxybenzyl diethylenetriamine pentaacetic acid-enhanced magnetic resonance imaging. Oncol Lett. 2019 ;18(3):2322-2329. doi: 10.3892/ol.2019.10557.

20. Xu P, Zeng M, Liu K, Shan Y, Xu C, Lin J. Microvascular invasion in small hepatocellular carcinoma: is it predictable with preoperative diffusion-weighted imaging? J Gastroenterol Hepatol. 2014;29(2):330-6. doi: 10.1111/jgh.12358.

21. Yacoub JH, Elsayes KM, Fowler KJ, et al. Pitfalls in liver MRI: Technical approach to avoiding misdiagnosis and improving image quality. J Magn Reson Imaging. 2019;49(1):41-58. doi: 10.1002/jmri.26343.

22. Dhanda S, Thakur M, Kerkar R, Jagmohan P. Diffusion-weighted imaging of gynecologic tumors: diagnostic pearls and potential pitfalls. Radiographics. 2014;34(5):1393-416. doi: 10.1148/rg.345130131.

23. Kim SY, Lee SS, Byun JH, et al. Malignant hepatic tumors: short-term reproducibility of apparent diffusion coefficients with breath-hold and respiratory-triggered diffusion-weighted MR imaging. Radiology. 2010;255(3):815-23. doi: 10.1148/radiol.10091706.

24. Braithwaite AC, Dale BM, Boll DT, Merkle EM. Short- and midterm reproducibility of apparent diffusion coefficient measurements at 3.0-T diffusion-weighted imaging of the abdomen. Radiology. 2009;250(2):459-65. doi: 10.1148/radiol.2502080849.

25. Andreou A, Koh DM, Collins DJ, Blackledge M. Measurement reproducibility of perfusion fraction and pseudodiffusion coefficient derived by intravoxel incoherent motion diffusion-weighted MR imaging in normal liver and metastases. Eur Radiol. 2013;23(2):428-34. doi: 10.1007/s00330-012-2604-1.

26. Ding Y, Rao S, Yang L, Chen C, Zeng M. Comparison of the effect of region-of-interest methods using gadoxetic acid-enhanced MR imaging with diffusion-weighted imaging on staging hepatic fibrosis. Radiol Med. 2016;121(11):821-827. doi: 10.1007/s11547-016-0669-7

27. Ding Y, Rao SX, Zhu T, Chen CZ, Li RC, Zeng MS. Liver fibrosis staging using T1 mapping on gadoxetic acid-enhanced MRI compared with DW imaging. Clin Radiol. 2015;70(10):1096-103. doi: 
10.1016/j.crad.2015.04.014.

28. Hoad CL, Palaniyappan N, Kaye $P$, et al. A study of $T \nabla$ relaxation time as a measure of liver fibrosis and the influence of confounding histological factors. NMR Biomed. 2015;28(6):706-14. doi: 10.1002/nbm.3299.

29. Pawlik TM, Delman KA, Vauthey JN, et al. Tumor size predicts vascular invasion and histologic grade: Implications for selection of surgical treatment for hepatocellular carcinoma. Liver Transpl. 2005;11(9):1086-92. doi: 10.1002/lt.20472

30. Ueno A, Masugi Y, Yamazaki K, et al. OATP1B3 expression is strongly associated with $\mathrm{Wnt} / \beta$-catenin signalling and represents the transporter of gadoxetic acid in hepatocellular carcinoma. J Hepatol. 2014;61(5):1080-7. doi: 10.1016/j.jhep.2014.06.008.

31. Cieply B, Zeng G, Proverbs-Singh T, Geller DA, Monga SP. Unique phenotype of hepatocellular cancers with exon-3 mutations in beta-catenin gene. Hepatology. 2009 Mar;49(3):821-31. doi: 10.1002/hep.22695.

\section{Tables}

Table 1. Baseline characteristics of patients with hepatocellular carcinoma

\begin{tabular}{|c|c|c|c|c|}
\hline \multirow[b]{2}{*}{ Characteristics } & \multirow[b]{2}{*}{$\begin{array}{l}\text { All patients } \\
(\mathrm{n}=94)\end{array}$} & \multicolumn{2}{|c|}{ MVI } & \multirow[b]{2}{*}{$P$} \\
\hline & & $\begin{array}{l}\text { Negative } \\
(n=56)\end{array}$ & $\begin{array}{l}\text { Positive } \\
(\mathrm{n}=38)\end{array}$ & \\
\hline Gender & & & & 0.217 \\
\hline Male & 88 & 54 & 34 & \\
\hline Female & 6 & 2 & 4 & \\
\hline Age, years (Range) & $52(24-75)$ & $52(30-73)$ & $53(24-75)$ & 0.744 \\
\hline Diameter, cm (Range) & $1.9(1.2-4.5)$ & $1.8(1.2-3.5)$ & $2.2(1.4-4.5)$ & 0.078 \\
\hline Etiology & & & & 0.190 \\
\hline $\mathrm{HBV}$ & 87 & 54 & 33 & \\
\hline $\mathrm{HCV}$ & 5 & 1 & 4 & \\
\hline Alcohol or others & 2 & 1 & 1 & \\
\hline $\mathrm{AFP}>20 \mathrm{ng} / \mathrm{ml}$ & & & & $>0.99$ \\
\hline Positive & 51 & 30 & 21 & \\
\hline Negative & 43 & 26 & 17 & \\
\hline Child-Pugh & & & & $>0.99$ \\
\hline A & 91 & 54 & 37 & \\
\hline B & 3 & 2 & 1 & \\
\hline
\end{tabular}

HBV: Hepatitis B virus; HCV: Hepatitis C virus; AFP: a-fetoprotein; MVI: microvascular invasion

Table 2. Comparisons of mean values and standard deviations of T1 relaxation time and apparent diffusion coefficient (ADC) value between MVI-negative and MVI-positive groups of hepatocellular carcinoma. 


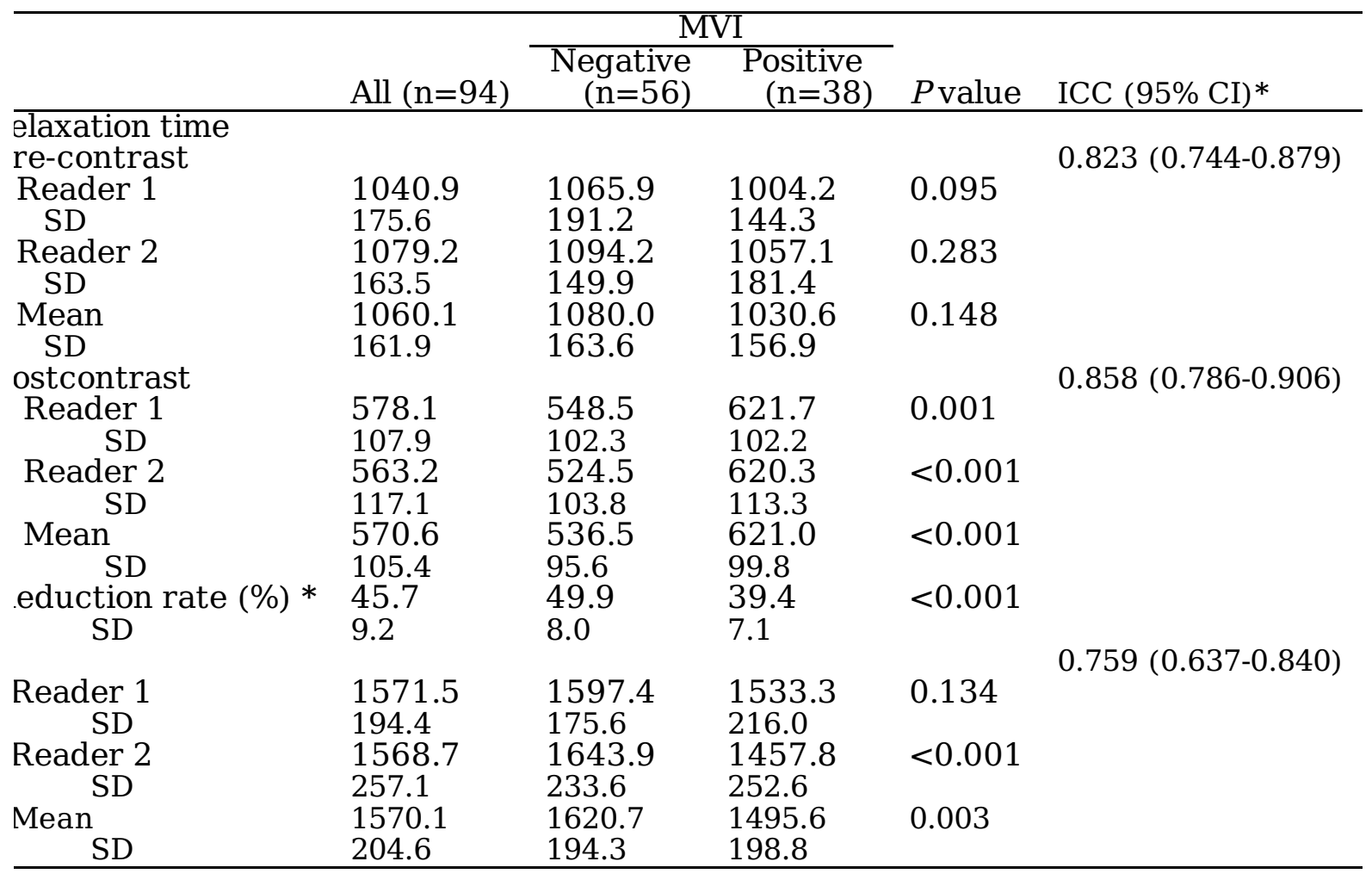

MVI: microvascular invasion; SD: standard deviations; CI: confidence intervals;

ICC: interclass correlation coefficient; ADC: apparent diffusion coefficient;

* The ICC was not calculated because reduction rate of T1 relaxation was based on the mean values of precontrast and postcontrast $\mathrm{T} 1$ relaxation time of the two readers.

Table 3. Diagnostic performance for T1 relaxation time and ADC value in evaluating MVIpositive HCC by receiver operating characteristic analyses.

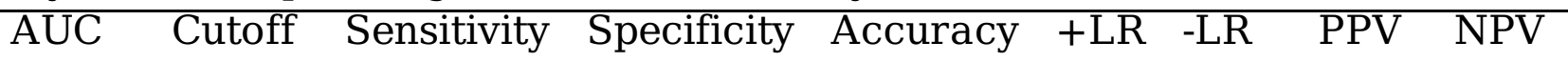

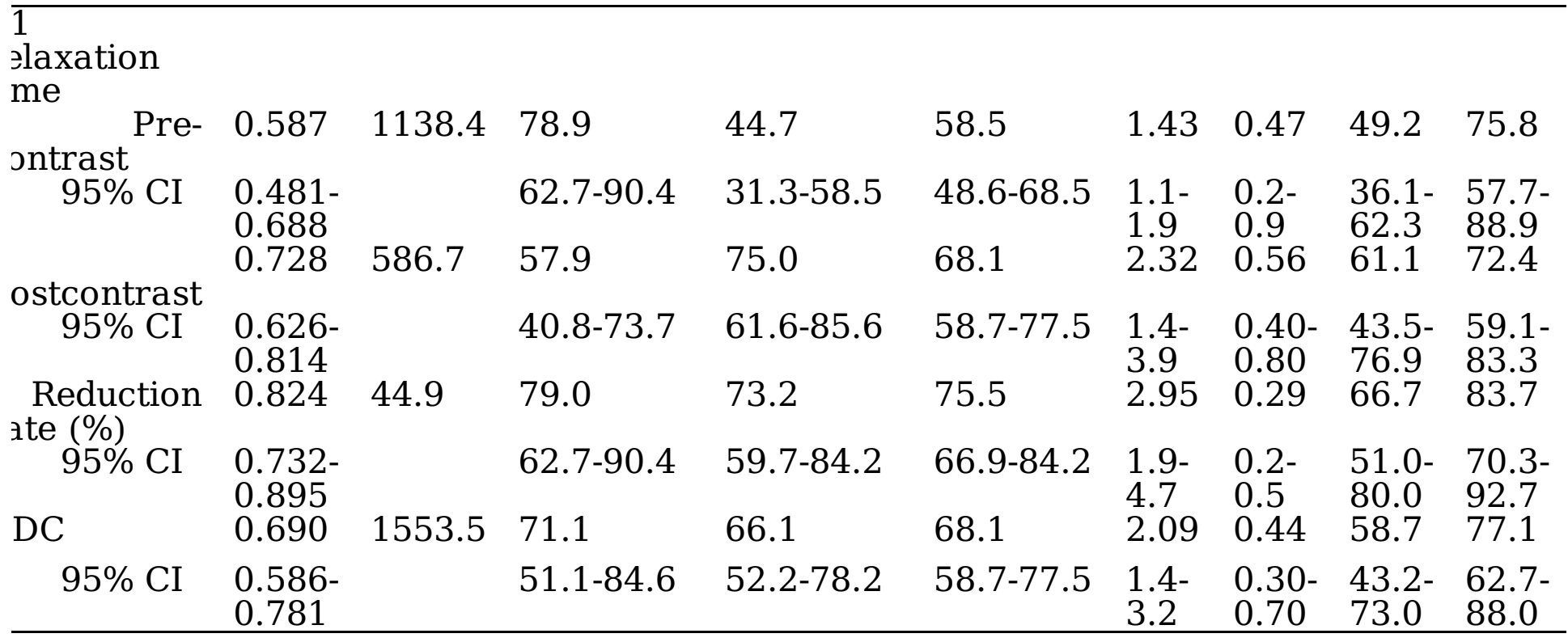

AUC: area under receiver operating characteristic curve; LR: likelihood ratio; PPV: positive predictive value; NPV: negative predictive value;

CI: confidence intervals; ADC: apparent diffusion coefficient; 


\section{2 patients with suspicious HCC underwent preoperative gadoxetic acid-enhanced MRI}

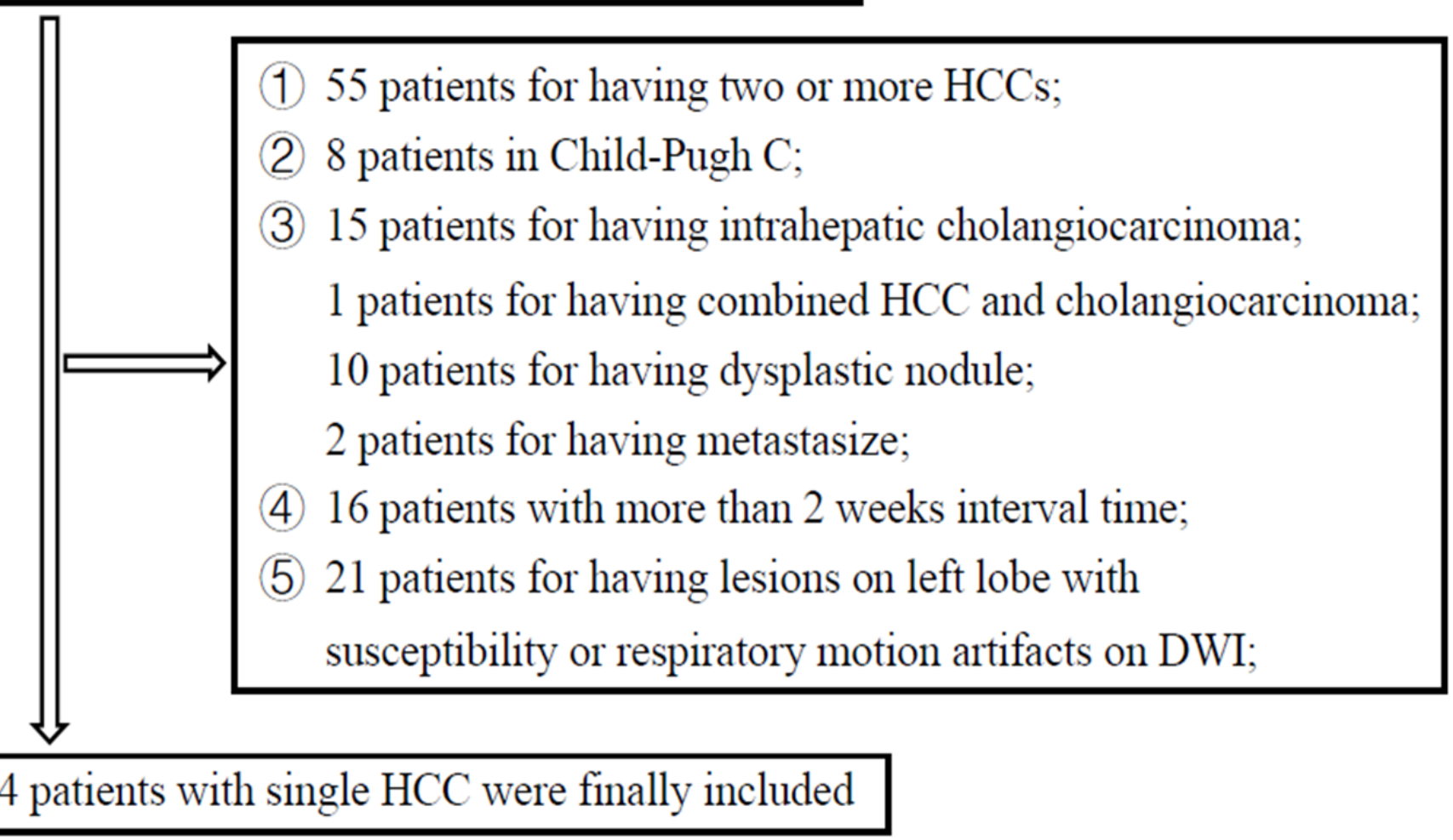

Figure 1

Flow chart of patients selection. 


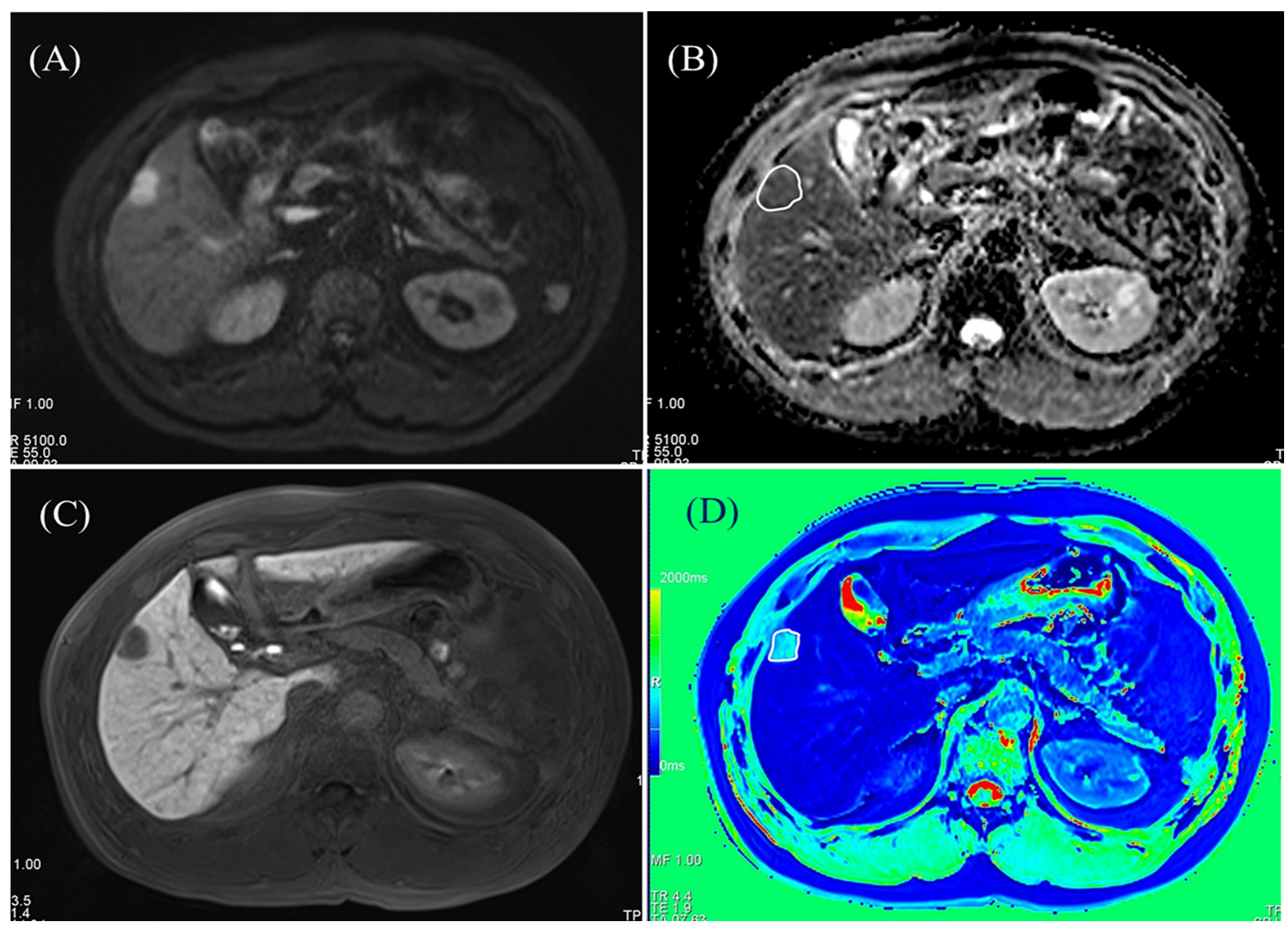

Figure 2

The ROls of HCC was drawn on the corresponding ADC maps and postcontrast T1 maps. (A) the HCC on right lobe of liver showing hyperintensity on DWI maps of $b=500 \mathrm{~mm} 2 / \mathrm{s}$; (B) ROI was drawn on corresponding ADC maps; $(C)$ the HCC on right lobe of liver showing hypointensity on hepatobiliary phase; (D) ROI was drawn on postcontrast T1 maps. 


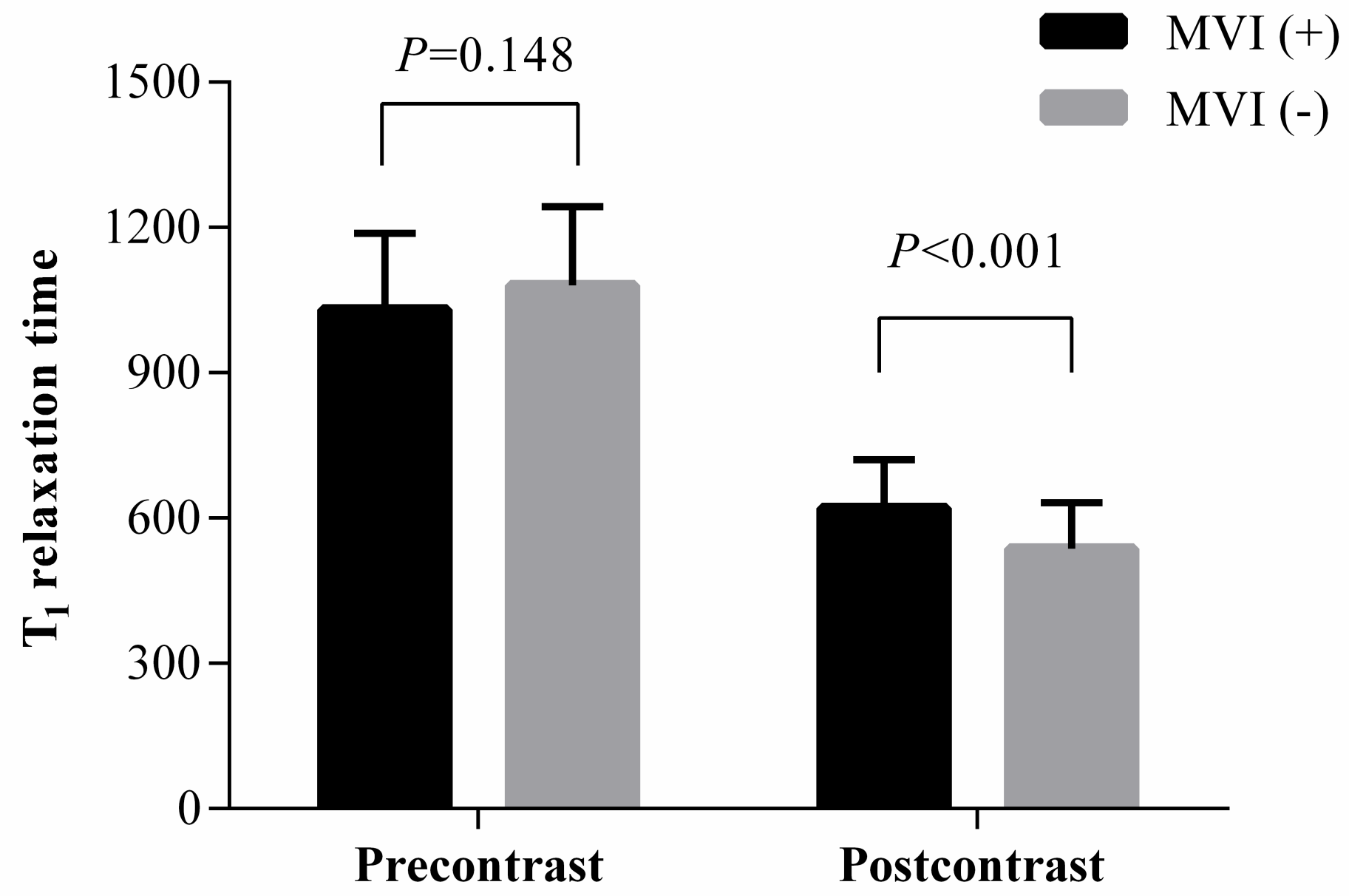

Figure 3

Comparisons of mean values and standard deviation of precontrast and postcontrast T1 relaxation time between MVI-positive and MVI-negative HCCs. 


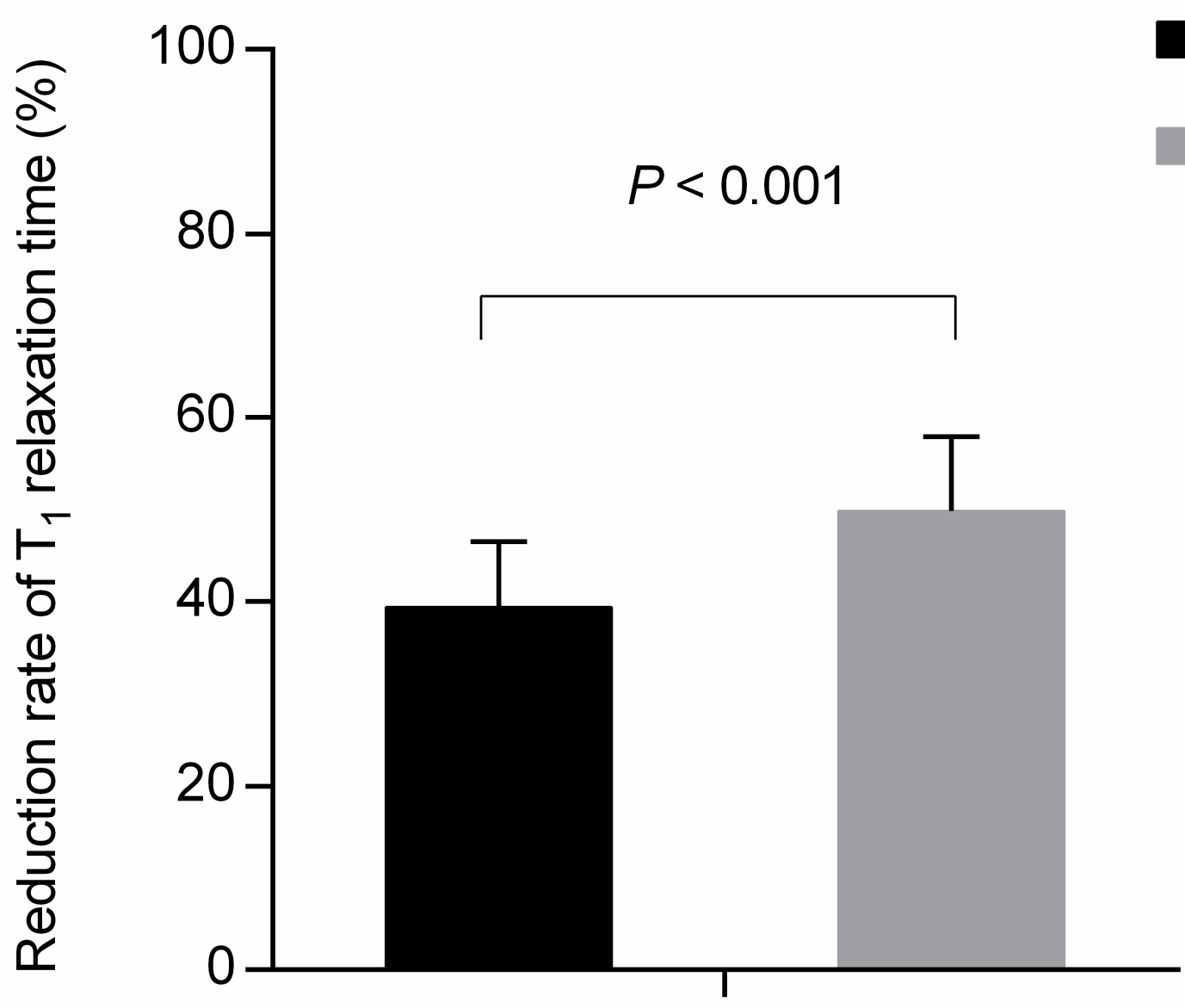

Figure 4

Comparisons of mean values and standard deviation of reduction rate T1 relaxation time between MVIpositive and MVI-negative HCCs. 


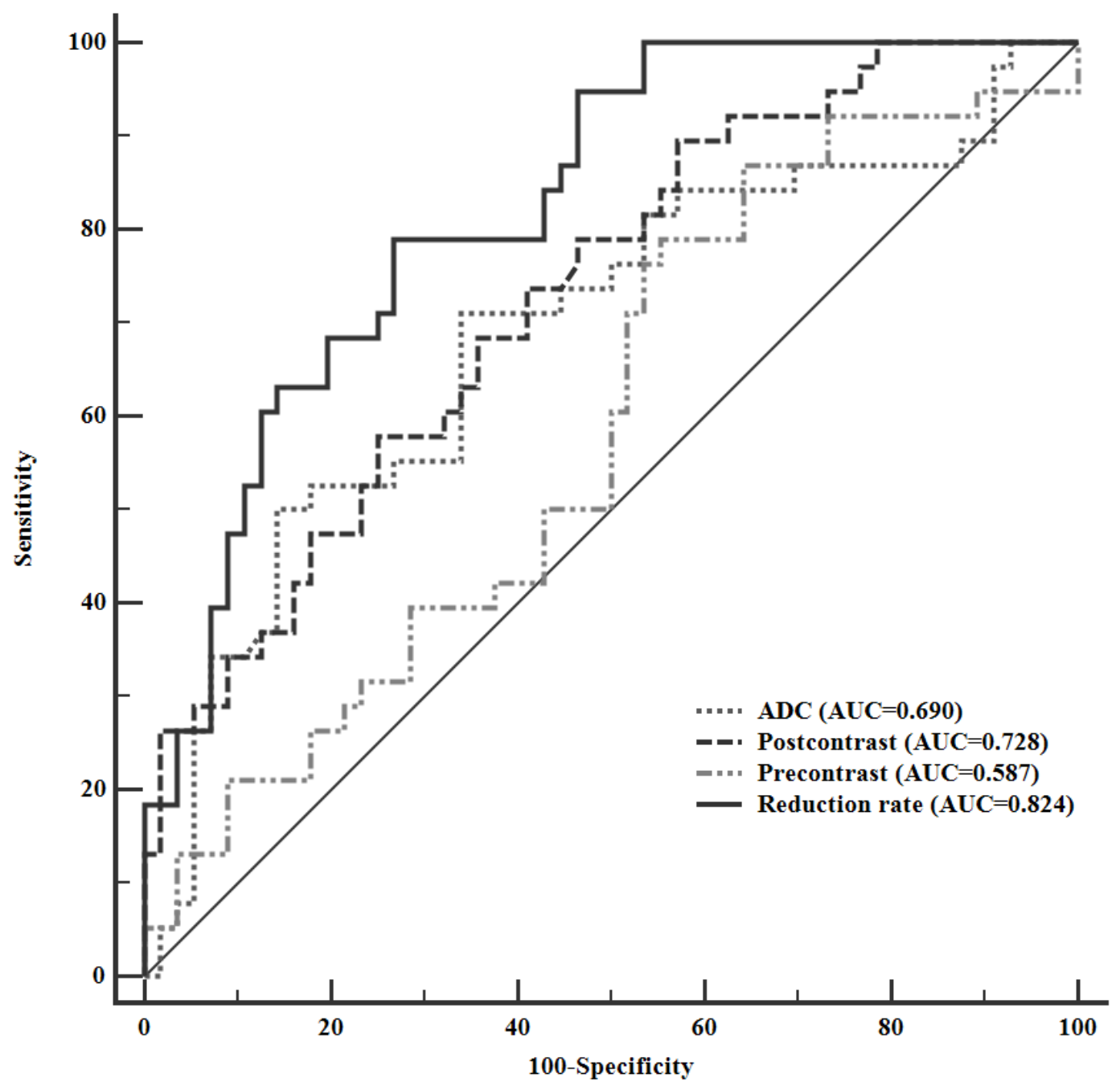

Figure 5

The utility of receiver operating characteristic curve of precontrst, postcontrast and reduction rate of T1 relaxation time and $A D C$ value to discriminate MVI-positive and MVI-negative HCCs. The AUC of reduction rate of $\mathrm{T} 1$ relaxation time was 0.801 with the optimal cutoff value of $44.9 \%$. 